\title{
Diagnostik und Therapie der Polycythaemia vera im Jahre 2015
}

\author{
Eva Lengfelder, Martin Griesshammer, Steffen Koschmieder
}

\begin{abstract}
Aktueller Stand
Die klassischen myeloproliferativen Neoplasien, chronische myeloische Leukämie, Polycythaemia vera (PV), essenzielle Thrombozythämie (ET) und die primäre Myelofibrose (PMF), wurden in der Vergangenheit aufgrund der klinischen Ähnlichkeit unter dem Begriff der chronischen myeloproliferativen Erkrankungen zusammengefasst [1]. Nach der Entdeckung des Philadelphia-Chromosoms und des korrespondierenden bcr-abl-Fusionsgens [2,3], welche eine klare Abgrenzung der CML und die Entwicklung einer zielgerichteten am molekularen Defekt ansetzenden Therapie erlaubten, standen die anderen Entitäten über längere Zeit im Hintergrund des klinischen und wissenschaftlichen Interesses. Erst im Jahre 2005 eröffnete die Entdeckung der JAK2V617F-Mutation, einer Punktmutation im Gen der JAK2-Tyrosinkinase, neue Perspektiven für Diagnostik und Therapie dieser Erkrankungen [4].
\end{abstract}

Die JAK2-V617F-Mutation wird nicht nur als klonaler Marker zur Diagnosesicherung von PV, ET, PMF und einzelner seltenerer Entitäten eingesetzt, sie ist insbesondere auch eine Zielstruktur für die Entwicklung selektiver Tyrosinkinaseinhibitoren (TKI). Mit der Zulassung des JAK1/JAK2Inhibitors Ruxolitinib (Jakavi ${ }^{\circledR}$ ) für Patienten mit $\mathrm{PV}$, die sich refraktär oder intolerant gegenüber Hydroxyurea zeigten, steht erstmals ein TKI für die Behandlung dieser Erkrankung zur Verfügung.

In Europa liegt die Inzidenz der PV zwischen 0,4\% und 2,8\% pro 100000 Einwohner pro Jahr [5]. Das mediane Alter bei Diagnosestellung beträgt etwa 60 Jahre [6, 7]. Die durchschnittliche Lebenserwartung liegt nach aktuellen Analysen bei knapp 19 Jahren und ist trotz dieser relativ günstigen Daten schlechter als die der Normalbevölkerung [6].

Der natürliche Krankheitsverlauf der PV umfasst unterschiedliche Stadien. Die in der Regel über Jahre anhaltende chronische Phase ist durch die klinischen Merkmale der gesteigerten Myeloproliferation charakterisiert. Diese imponiert als Proliferation aller drei Zellreihen (Erythropoese, Megakaryopoese, Granulopoese) und Splenomegalie. Die Proliferation der Erythropoese steht hierbei im Vordergrund und bestimmt das klinische Bild. Häufigste und potenziell bedrohliche Komplikationen sind arterielle oder venöse Thromboembolien bei bis zu $40 \%$ der Patienten [6, 7]. Bei unbehandelter PV stellen sie mit $63 \%$ der

\section{Was ist neu?}

- Neue Entwicklungen bei Diagnosesicherung und Pathogenese: Hauptkriterien der zur Zeit noch aktuellen WHO-Klassifikation der Polycythaemia vera (PV) sind eine gesteigerte Erythrozytenmasse und der Nachweis eines klonalen Markers (JAK2-Mutation).

- Klinische Symptome: Der Schweregrad der PV-Symptome ist häufig moderat, jedoch leiden viele Patienten an mehreren Symptomen, wodurch die Lebensqualität eingeschränkt ist.

- Therapieziele und Risikostratifizierung: Bei in der Regel guter Überlebensprognose ist die Reduktion der potenziell bedrohlichen thromboembolischen Komplikationen primäres Therapieziel. Das Überleben wird signifikant von der Anzahl der vorliegenden Risikofaktoren beeinflusst.

- Therapie: Aderlass, ASS und zytoreduktive Therapie (Hydroxyurea bzw. Interferon-alpha) sind die Eckpfeiler der Therapie. Der TyrosinkinaseInhibitor Ruxolitinib erweitert die Therapiemöglichkeiten bei einer Resistenz und Intoleranz gegenüber Hydroxyurea.

Todesfälle die häufigste Todesursache dar [8]. Probleme der Spätphase sind der Übergang in eine sekundäre Myelofibrose (MF) mit extramedullärer Hämatopoese und zum Teil ausgeprägter Splenomegalie und die Transformation in eine akute Leukämie. Die Rate an MF liegt nach einer medianen Beobachtungszeit von 10 Jahren bei $15 \%$, nach 20 Jahren bei 50\%. Der direkte Übergang in eine akute Leukämie ist selten (4\%), während etwa $20 \%$ der Patienten mit Post-PV MF in eine AML transformieren [6, 7, 10-12].

\section{Neuere Entwicklungen bei Diagnosesicherung und Pathogenese}

Die Diagnose der PV wird derzeit noch auf der Basis der WHO-Kriterien aus dem Jahr 2008 gestellt ( Tab. 1) [13]. Diese Klassifikation schließt ge-

Tab. 1 Diagnosekriterien der WHO von 2008 [13]. Die Diagnose Polycythaemia vera wird gestellt, wenn beide Hauptkriterien und ein Nebenkriterium oder wenn das erste Hauptkriterium und zwei Nebenkriterien vorliegen.
Hauptkriterien
- Hämoglobin $>18,5 \mathrm{~g} / \mathrm{dl}$ bei Männern, $>16,5 \mathrm{~g} / \mathrm{dl}$ bei Frauen oder andere Evidenz einer gesteigerten Erythro- zytenmasse
- Nachweis der JAK2V617F-Mutation oder einer anderen funktionell ähnlichen Mutation (z. B. JAK2-Mutation im Exon 12)
Nebenkriterien - Hyperzellularität des Knochenmarkes mit gesteigerter Erythropoese, Granulopoese und Megakaryopoese
- Niedriger Erythropoetin-Spiegel
- Nachweis von endogenen erythroiden Kolonien in vitro 


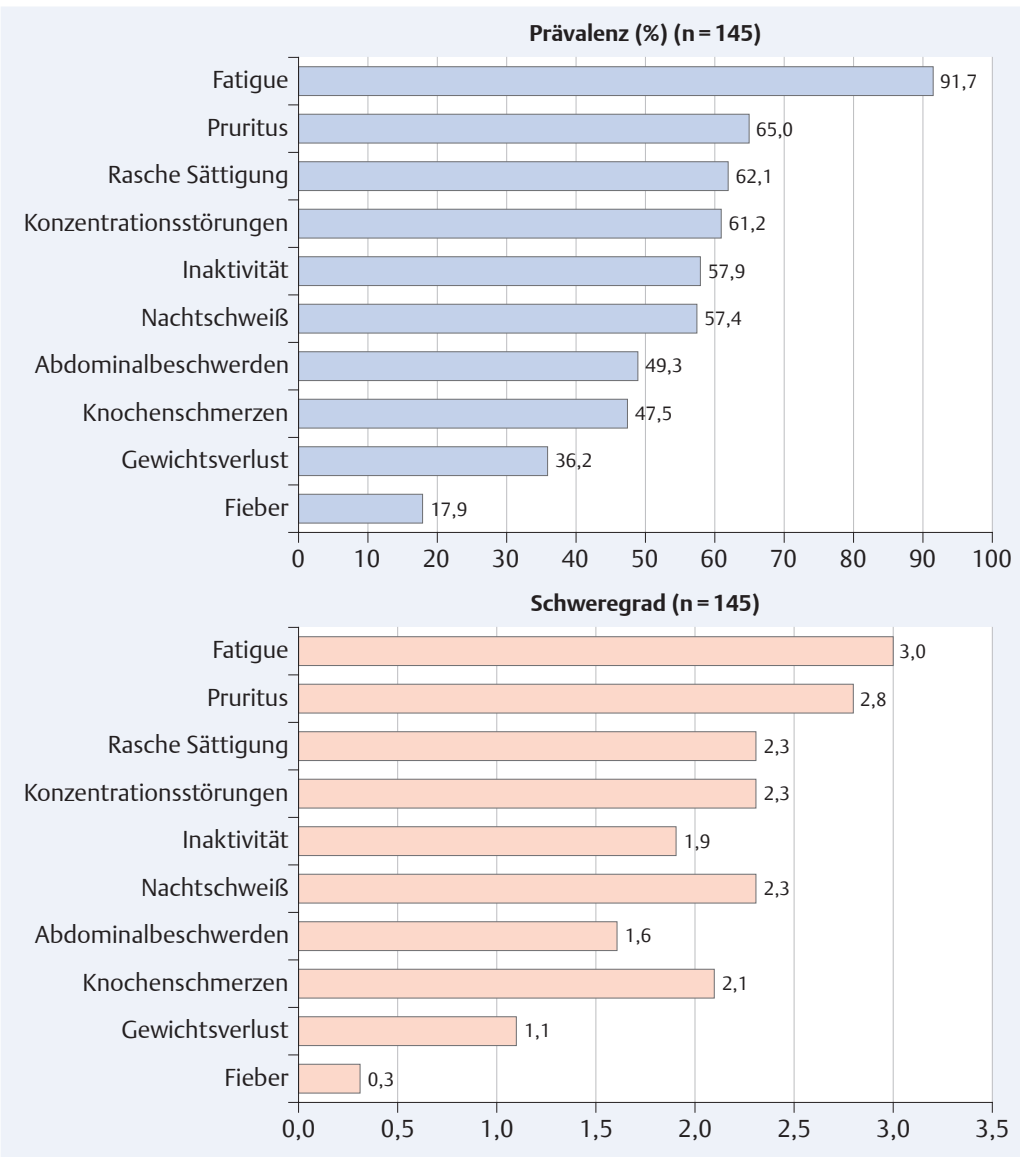

Abb. 1 Prävalenz und Schweregrad der Polycythaemia-vera-Symptome [29]. Der Schwergrad der Syptome wird nach einer Skala von Grad 1 bis 10 vom Patienten bewertet.

Tab. 2 Spektrum typischer klinischer Symptome bei Polycythaemia vera $[7,29]$. niebildung aus der Liste der Nebenkriterien in Diskussion [16].

Bei fehlendem Nachweis eines molekularen Markers (JAK2V617F- oder Exon12-Mutation) ist, wie bereits in der Vergangenheit, differenzialdiagnostisch eine sekundäre Polyglobulie auszuschließen. Häufigere Ursachen hierfür sind kardiale oder pulmonale Erkrankungen, insbesondere Herzinsuffizienz und chronische Lungengerüsterkrankungen sowie starker Nikotinkonsum. Bei der Suche nach selteneren Ursachen ist insbesondere an das Schlaf-Apnoe-Syndrom, Tumorerkrankungen mit paraneoplastischer Erythropoietinproduktion, medikamentös induzierte Polyglobulie und kongenitale Erythrozytosen zu denken [17, $18]$.

Nach aktuellem Kenntnisstand kommt Mutationen, die den JAK-STAT-Signalweg betreffen, eine Schlüsselrolle in der Pathogenese verschiedener Malignome oder durch Veränderungen im Immunsystem ausgelöster Erkrankungen zu [19, 20]. Neben den Mutationen im JAK-STAT-Signalweg wurden zusätzlich weitere, auf anderen Ebenen der Signaltransduktion und Genregulation ansetzende Mutationen gefunden [21]. Für verschiedene Entitäten der MPN, einschließlich der $\mathrm{PV}$, konnte gezeigt werden, dass eine höhere Anzahl an Mutationen signifikant mit einer schlechteren Prognose korreliert [22]. Ergebnisse aus jüngster Vergangenheit machten deutlich, dass die Reihenfolge des Auftretens der Mutationen (z.B. von TET2 und JAK2V617F) einen Einfluss auf klinische Parameter, z. B. den Phänotyp (PV vs. ET) und die Empfindlichkeit der Zellen gegenüber Ruxoltinib haben kann [23]. Die zunehmend bessere Kenntnis der molekularen Veränderungen bei den MPN einschließlich der PV und deren Korrelation zur Prognose deutet darauf hin, dass das individuelle molekulare Profil in Zukunft einen wichtigen Stratifizierungsparameter für Therapieentscheidungen darstellen dürfte.

\section{Klinische Relevanz}

Das Screening auf JAK2-Mutationen ist bei bestehendem Verdacht auf PV obligat.
Arterielle und venöse Thromboembolien im
Mikrozirkulationsstörungen
Konstitutionelle und allgemeine Symptome Bereich der großen Gefäße
- Myokardinfarkt
- Akutes Koronarsyndrom
- Apoplex, TIA
- Periphere arterielle Verschlusskrankheit
- Tiefe Venenthrombose
- Lungenarterienembolie
- Intraabdominelle Venenthrombose (Budd-
- Müdigkeit (Fatigue)
- Pruritus
- Nachtschweiß
- Knochenschmerzen
- Fieber
- Splenomegalie
- Gewichtsverlust Chiari-Syndrom, Pfortaderthrombose u.a.)

- Zerebralsinus-Thrombose 


\section{Klinische Symptome}

Im früheren Krankheitsstadium stehen Symptome im Vordergrund, die durch die erhöhte Blutviskosität bedingt sind. Zu den thromboembolischen Komplikationen trägt neben dem erhöhten Hämatokrit und der ggf. gesteigerten Thrombozytenzahl eine ganze Reihe inzwischen recht gut definierter weiterer thrombogener Mechanismen bei, die eine zusätzliche Aktivierung von Gefäßendothel, Gerinnungssystem, Leukozyten und Thrombozyten bewirken können [24]. Relativ häufig sind kardiale und zerebrale arterielle Gefäßverschlüsse und periphere Venenthrombosen. Selten, aber für die PV und andere MPN nicht ungewöhnlich, sind abdominelle Venenthrombosen (Budd-Chiari-Syndrom, Pfortaderthrombose, Nierenvenenthrombose u.a.) und Thrombosen im Cerebralsinus [25-28]. Auch Mikrozirkulationsstörungen führen zu charakteristischen klinischen Symptomen ( $\bullet$ Tab. 2).

In aktuellen Analysen aus den USA und Europa wurde anhand von Patientenfragebögen Häufigkeit und Schweregrad der klinischen Symptome (Grad 1 bis 10) der Patienten erfasst ( Abb. 1). Die Resultate machen deutlich, dass der durchschnittliche Schweregrad zwar relativ moderat ist, dass aber nicht wenige Patienten an mehreren Symptomen leiden, was die Lebensqualität erheblich beeinflussen kann $[29,30]$. Insbesondere der oft quälende und nicht selten therapierefraktäre Pruritus stellt für manche Patienten ein die Lebensqualität und den Aktionsradius massiv beeinträchtigendes Symptom dar. Die systematische Erfassung der mit MPN assoziierten Symptome und deren Ausprägung haben wesentlich dazu beigetragen den ärztlichen Blick für diese Problematik zu schärfen.

\section{Klinische Relevanz}

Patienten mit PV haben ein breites Spektrum an Symptomen, welche die Lebensqualität erheblich einschränken können.

\section{Therapieziele und Risikostratifizierung}

Vordringliche Therapieziele bei der PV sind

- die Vermeidung thromboembolischer Komplikationen und

- die Besserung der subjektiven Beschwerden.

- Vermeidung von Spätkomplikationen

In erster Linie soll die Therapie thromboembolische Komplikationen reduzieren. Sie wird in Abhängigkeit vom Thromboserisiko stratifiziert [17, 31]. Höheres Alter und bereits stattgehabte thromboembolische Komplikationen sind entscheidende Risikofaktoren für Thrombosen und die Basis der Risikostratifizierung. Bei jüngerem Lebensalter (unter 60 Jahren) ohne weitere Risikofaktoren

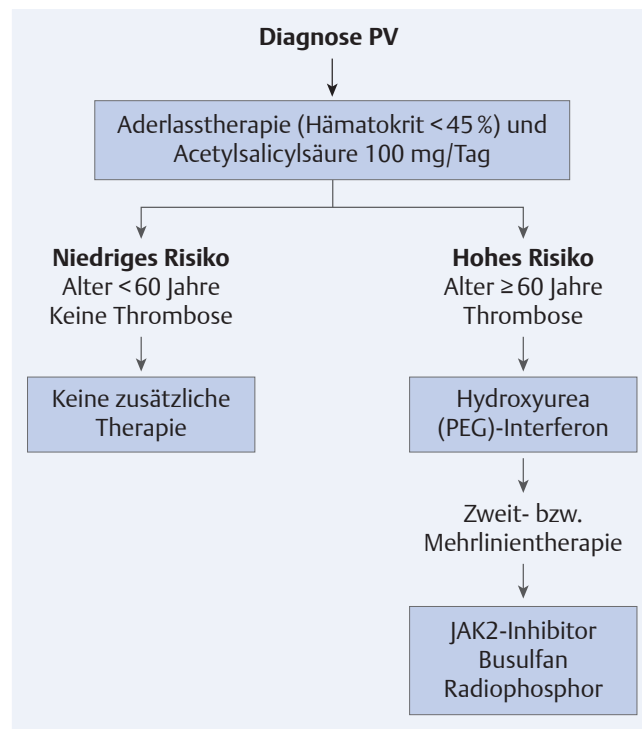

Abb. 2 Therapiealgorithmus bei Polycythaemia vera (PV). Interferon wird vorzugsweise bei jüngeren Patienten (<40 Jahren) empfohlen, Busulfan und Radiophosphoe bei älteren Patienten (> 75 Jahren) [17,31].

liegt ein niedriges Thromboembolierisiko vor, bei Vorhandensein eines oder beider Faktoren ein hohes Risiko. Auch bei Niedrigrisikopatienten ist das Thromboembolierisiko verglichen mit der Normalbevölkerung etwa um den Faktor 2,5 erhöht, liegen beide Risikofaktoren vor ist es 10 -fach höher [32]. Weitere Risikofaktoren sind kardiovaskuläre Erkrankungen, Nikotinkonsum sowie eine hohe Leukozytenzahl, die bei Entscheidungssituationen berücksichtigt werden sollten [33].

In jüngerer Vergangenheit wurden zur Einschätzung der individuellen Überlebensprognose von PV-Patienten Scores entwickelt. Beide Scores wurden auf Basis retrospektiver Datensammlungen zusammengestellt. Sie schließen höheres Lebens-

Tab. 3 ELN-Definition der Resistenz oder Intoleranz gegenüber Hydroxyurea (HU) bei Polycythaemia vera [41].

1. Aderlassbedürftigkeit nach 3-monatiger Therapie mit mindestens $2 \mathrm{gHU} / \mathrm{Tag}$, um den Hämatokrit unter $45 \%$ zu halten oder

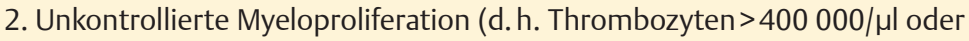
Leukozyten> 10 000/ $\mu$ l) nach 3 Monaten Therapie mit mindestens $2 \mathrm{~g} \mathrm{HU} / \mathrm{Tag}$ oder

3. Milzgrößenreduktion unter $50 \%$ bei massiver $^{1}$ Splenomegalie (Beurteilung durch Palpation) oder unvollständiges Verschwinden von durch die Splenomegalie bedingten Symptomen nach 3-monatiger Therapie mit mindestens $2 \mathrm{~g}$ $\mathrm{HU} / \mathrm{Tag}$ oder

4. Absolute Neutrophilenzahl $<1000 / \mu$ l oder Thrombozytenzahl $<100000 / \mu l$ oder Hämoglobin $<10 \mathrm{~g} / \mathrm{dl}$ mit der niedrigsten Dosis von HU, die erforderlich ist, um ein komplettes ${ }^{2}$ oder partielles ${ }^{3}$ klinisch-hämatologisches Ansprechen zu erzielen oder

5. Ulzera an den Beinen oder andere unakzeptable HU-bedingte nicht-hämatologische Toxizitäten, wie andere Manifestationen an Haut oder Schleimhäuten, gastrointestinale Symptome, Pneumonitis oder Fieber unabhängig von der Dosierung von HU.

${ }^{1}$ Milz> $10 \mathrm{~cm}$ unter dem Rippenbogen

${ }^{2}$ Hämatokrit $<45 \%$ ohne Aderlasstherapie, Thrombozyten $\leq 400000 / \mu \mathrm{l}$, Leukozyten, $\leq 10000 / \mu$ und keine krankheitsbedingten Symptome

${ }^{3}$ Hämatokrit< $45 \%$ ohne Aderlasstherapie oder Ansprechen von mehr als drei anderen Kriterien 
Hämatologisches Ansprechen

- Kombinierter primärer Endpunkt ${ }^{1}$

- Hämatokritkontrolle

- Reduktion des Milzvolumens

- Komplettes hämatologisches Ansprechen 2,3

Symptomkontrolle

- $50 \%$ Reduktion im Gesamtsymptom-Score ${ }^{4}$

$49 \%$

$\begin{array}{ll}21 \% & 1 \% \\ 60 \% & 20 \% \\ 38 \% & 1 \% \\ 24 \% & 9 \%\end{array}$

Mediane Zu- oder Abnahme im Symptom-Score (Therapiebeginn bis Woche 32)

- Müdigkeit

- Juckreiz

- Nachtschweiß

- Schwindelgefühl

- Konzentrationsstörungen

- Rasche Sättigung

- Abdominelle Beschwerden

${ }^{1}$ Kombinierter Endpunkt: Hämatokritkontrolle (nicht mehr als ein Aderlass zwischen Therapiebeginn und Woche 8 , keine Aderlassbedürftigkeit zwischen Woche 8 und 32) und Reduktion des Milzvolumens $\geq 35 \%$,

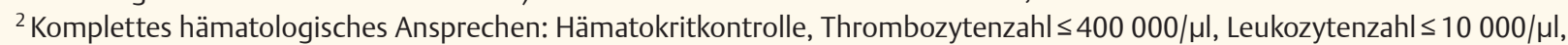

${ }^{3} \mathrm{p}=0,003$

${ }^{4}$ verwendete Symptom-Scores siehe Originalpublikation [51]

Tab. 4 Auswahl wichtiger Resultate mit Ruxolitinib im Vergleich zu bester verfügbarer Therapie [51]. alter (>60 bzw. > 70 Jahre), eine zum Diagnosezeitpunkt stattgehabte Thrombose und eine erhöhte Leukozytenzahl (>13000/ $\mathrm{ll}$ bzw. $\geq 15000 / \mu \mathrm{l}$ ) als Risikofaktoren ein. Das Überleben von PV-Patienten wird signifikant von der Anzahl dieser Faktoren beeinflusst; Patienten ohne Riskofaktor hatten die beste und Patienten mit gleichzeitigem Vorliegen mehrerer Risikofaktoren die schlechteste Prognose [6, 34].

\section{Klinische Relevanz}

Die Senkung des Thromboembolierisikos ist ein wesentliches Ziel bei der Therapie der PV.

\section{Therapie - aktueller Stand}

Der aktuell empfohlene Therapiealgorithmus ( $\triangleright$ Abb. 2) entspricht den internationalen Therapieempfehlung für PV $[17,33,35]$. Nachdem in der Vergangenheit der Zielhämatokrit unzureichend definiert war und die Therapie mit Acetylsalicylsäure (ASS) wegen des gefürchteten Blutungsrisikos bei höheren Dosen als obsolet galt, gibt es inzwischen für den Einsatz beider Therapiemaßnahmen aufgrund randomisierter Studien klare Empfehlungen. Unabhängig von der individuellen Risikozuordnung ist das primäre Therapieziel die gleichmäßige Absenkung des Hämatokrit auf Werte unter $45 \%$ zunächst mit Aderlasstherapie, da höhere Grenzwerte mit einer gesteigerten Rate an arteriellen und venösen Thromboembolien verbunden waren [36]. Aufgrund des bei PV gesicherten antithrombotischen Effektes wird zusätzlich die Gabe von niedrig dosierter Azetylsalicylsäure (100 mg ASS pro Tag) empfohlen [37].

Für Patienten der Niedrigrisikogruppe gilt die Kombination von Aderlässen und ASS als Therapie der Wahl. Bei Patienten mit primär hohem Risiko besteht primär die Indikation zur zusätzlichen Einleitung einer zytoreduktiven Therapie, ggf. zunächst unter Fortsetzung der Aderlasstherapie bis zur Stabilisierung des Hämatokritwertes. Auch die im Verlauf zunehmende Krankheitsaktivität (Steigerung der Myeloproliferation mit zunehmender Aderlassfrequenz, Anstieg von Thrombozyten und Leukozyten und Zunahme der Splenomegalie, verstärktes Auftreten von konstitutionellen Symptomen u.a.) ist in der Regel eine Indikation zur Einleitung einer zytoreduktiven Therapie. Liegen zusätzlich kardiovaskulärer Risikofaktoren vor - bei ansonsten niedrigem Thromboserisiko - kann ebenfalls eine zytoreduktive Therapie indiziert sein. Genau definierte verbindliche Kriterien, wann eine zytoreduktive Therapie eingeleitet werden sollte, liegen allerdings nicht vor, sodass diese Entscheidung individuell getroffen werden muss. Die sehr begrenzt verfügbaren Resultate von Langzeitanalysen zeigten, dass der Anteil von Patienten, die 
über große Zeiträume mit einer alleinigen Aderlassbehandlung ausreichend therapierbar waren, unter $10 \%$ lagen [38].

Aufgrund des leukämogenen Effektes verschiedener in der Vergangenheit eingesetzter Substanzen (Alkylanzien, hier insbesondere Chlorambucil, sowie Radiophosphor) sind die verbliebenen zytoreduktiven Therapiemöglichkeiten bei der PV relativ begrenzt [39]. Hydroxyurea (HU) ist die aktuelle Standardtherapie zur Reduktion der Myeloproliferation. Auch für HU wurde primär ein leukämogenes Potenzial angenommen. Allerdings wurde dieser Effekt in prospektiven Studien nie eindeutig belegt, sodass er inzwischen, wenn überhaupt vorhanden, als sehr gering eingeschätzt wird [12, 32, 40]. Da Richtlinien für einen einheitlichen Gebrauch von HU fehlten, wurden im Rahmen des Europäischen Leukämienetzes Kriterien für Resistenz und Intoleranz gegenüber HU definiert ( $\bullet$ Tab. 3) [41]. In einer retrospektiven Anwendung dieser Kriterien auf ein Kollektiv von 261 PV-Patienten ergab sich, dass $12 \%$ der Patienten primär resistent und $13 \%$ intolerant waren. Etwa 40\% verloren das Ansprechen während des Therapieverlaufes. Zum Teil wurde die HUTherapie trotz unbefriedigenden Ansprechens beibehalten, was am ehesten auf den Mangel an therapeutischen Alternativen zurückgeführt werden kann. Es ist hervorzuheben, dass die Resistenz gegen HU mit einer schlechteren Überlebensprognose und einer höheren Transformationsrate in eine akute Leukämie verbunden war [42].

Interferon alpha (IFN) ist in herkömmlicher und in pegylierter Form verfügbar, jedoch für die bcrabl-negative MPN nicht zugelassen. Die Gesamtansprechrate bei PV beträgt etwa $80 \%$, die Rate an Aderlassfreiheit etwa 60\%. IFN ist nicht leukämogen und gilt deshalb als Therapie der Wahl bei jüngeren PV-Patienten. IFN kann bei einem nennenswerten Anteil von Patienten den JAK2V617F-positiven Klon bis unterhalb der Nachweisgrenze reduzieren [43-45]. Man geht deshalb davon aus, dass IFN auch die Entwicklung der Spätstadien (Myelofibrose und akute Leukämie) entweder ganz verhindern oder zumindest stark reduzieren kann.

Busulfan ist eine weitere Therapiemöglichkeit bei PV. Es wurde in früheren Therapiestudien eingesetzt, erwies sich als wenig toxisch und ist auch bei Patienten mit PV hochwirksam, die gegenüber mehreren anderen Substanzen therapierefraktär waren. Der leukämogene Effekt wird als gering eingeschätzt [46]. Busulfan stellt vor allem für ältere Patienten eine Therapiemöglichkeit dar, wenn andere Alternativen ausgeschöpft sind [47]. Die bislang einzige kurative Therapieoption, die allogene Knochenmark- bzw. Stammzelltransplantation, ist aufgrund der zumeist guten Prognose der PV in der Regel nur bei Übergang in eine Post-PV MF indiziert.

\section{Unerwünschte Effekte bis} Woche 32

Thromboembolien*

Diarrhoe*

Muskelkrämpfe*

Dyspnoe

Dyspnoe Grad 3 oder 4

Infektionen gesamt

Infektionen Grad 3 oder4

Herpes Zoster

Myelofibrose

AML

* Unerwünschte Effekte Grad 3 oder 4 nur in ganz vereinzelten Fällen.

\section{Neue Therapieoptionen}

Tyrosinkinaseinhibitoren (TKI) stellen eine neue Substanzgruppe zur Therapie von MPN dar. In den letzten Jahren wurden verschiedene Inhibitoren der Janus-Kinase (JAK) entwickelt [48]. Die JAKInhibitoren blockieren die ATP-Bindungsstelle der Tyrosinkinasedomäne und verhindern auf diese Weise die Phosphorylierung und damit Aktivierung der Kinase und der nachfolgenden Signalkaskaden (u. a. von STAT-Molekülen, welche als Transkriptionsfaktoren die Zellproliferation induzieren). Auf diese Weise wird die durch die JAK2V617F-Mutation induzierte konstitutionelle Aktivierung des JAK-STAT-Signalweges blockiert. Die bisher entwickelten JAK-Inhibitoren sind nicht ausschließlich auf die mutierte JAK2-Tyrosinkinase ausgerichtet, sondern hemmen auch den JAK2-Wildtyp und zumeist noch weitere Kinasen (z. B. JAK1, JAK3, TYK u.a) [17,18].

Die Wirksamkeit des selektiven JAK1/JAK2-Inhibitors Ruxolitinib wurde zunächst bei Myelofibrose untersucht und zeigte hier eine eindrucksvolle Rückbildung der oft exzessiven Splenomegalie und der konstitutionellen Symptome, was zur Zulassung der Substanz bei der MF führte $[49,50]$. Seit Kurzem sind auch Resultate einer Phase-IIIStudie bei PV verfügbar. In dieser Studie wurde der TKI mit der besten verfügbaren Therapie (,best available therapy“ - BAT) verglichen. Eingeschlossen wurden PV-Patienten, die (nach den modifizierten ELN-Kriterien [41]) resistent oder intolerant gegenüber HU waren ( $\downarrow$ Tab. 3). Weitere Voraussetzungen waren Aderlassbedürftigkeit und Splenomegalie. Die Therapie mit Ruxolitinib $(n=110)$ erwies sich hinsichtlich der Kontrolle der hämatologischen Parameter und der Besserung der klinischen Symptome der BAT $(n=112)$ als
Tab. 5 Auswahl relevanter unerwünschter Effekte unter Ruxolitinib im Vergleich zu bester verfügbarer Therapie [51]. 


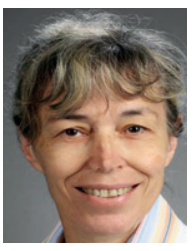

Prof. Dr. med.

Eva Lengfelder

ist Oberärztin der III.

Medizinischen Klinik,

Hämatologie und Onkologie am Universitätsklinikum

Mannheim der Universität Heidelberg.

eva.lengfelder@umm.de

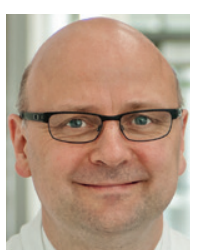

Prof. Dr. med.

Martin Grieshammer

ist Chefarzt der Klinik für

Hämatologie, Onkologie,

Hämostaseologie und

Palliativmedizin am Johannes

Wesling Klinikum Minden.

martin.griesshammer@

muehlenkreiskliniken.de

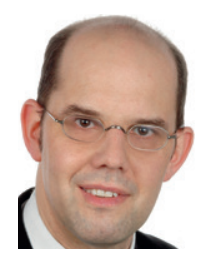

Prof. Dr. med.

Steffen Koschmieder

ist Leiter des Lehr- und

Forschungsgebiets

Translationale Hämatologie und Onkologie und Oberarzt der Klinik für Hämatologie, Onkologie, Hämostaseologie und Stammzelltransplantation an der Uniklinik RWTH

Aachen.

skoschmieder@ukaachen.de

DOI 10.1055/s-0041-103336

Dtsch Med Wochenschr 2015; 140: 1501-1506

(c) Georg Thieme Verlag KG . Stuttgart · New York .

ISSN 0012-0472 eindeutig überlegen und belegte somit die Wirksamkeit dieser Substanz auch bei der PV [51].

Die Hauptresultate der Studie sind in $\downarrow$ Tab. 4 dargestellt. Die Überlegenheit des Ruxolitinib-Armes betraf das Erreichen des primären Endpunktes (20,9\% vs. 0,9\%; p<0,001), definiert als Hämatokritkontrolle und Reduktion des Milzvolumens (geforderte Abnahme $\geq 35 \%$ im MRT) in Woche 32 nach Therapiebeginn. Hämatokritkontrolle lag vor, wenn maximal ein Aderlass bis Woche 8 und kein Aderlass zwischen Woche 8 und 32 notwendig war. Noch eindrucksvoller waren die Vorteile von Ruxolitinib gegenüber BAT bei der Einzelauswertung der beiden Parameter (Hämatokritkontrolle: $60 \%$ vs, 19,6\%; Milzvolumen: 38,2\% vs. $0,9 \%$ ). Ein komplettes hämatologisches Ansprechen hatten 23,6\% der Patienten der RuxolitinibGruppe vs. 8,9\% der BAT-Gruppe ( $\mathrm{p}=0,003)$. Weitere Vorteile für Ruxolitinib ergaben sich im Hinblick auf den Rückgang der Allel-Last und der niedrigeren Rate an thromboembolischen Komplikationen [51].

Die Therapie mit Ruxolitinib war auch mit einer eindrucksvollen Besserung der klinischen Symptome verbunden ( $\bullet$ Tab. 4 ). Besonders hervorzuheben ist die hohe Rückbildungsrate des oft therapierefraktären Pruritus. Die Daten weisen außerdem auf die Nachhaltigkeit der Effekte von Ruxolitinib hin, da 84,5\% der Patienten nach einem medianen Follow up von 81 Wochen weiterhin Ruxolitinib einnahmen, während 96,4\% der Patienten aus dem BAT-Arm auf Ruxolitinib gewechselt waren. Das schlechtere Abschneiden des BATArmes ist allerdings nicht ganz unerwartet, da 59\% der Patienten im BAT-Arm HU erhielten, auf welches sie als resistent oder intolerant eingestuft worden waren [49]. Wie bereits bei der Myelofibrose gezeigt, hat Ruxolitinib eine geringe Toxizität ( Tab. 5). Zu erwähnen ist die etwas höhere Rate an Infektionen im Ruxolitinib-Arm, möglicherweise als Ausdruck immunsuppressiver und antiinflammatorischer Effekte [51].

Von einer weiteren in Auswertung befindlichen Studie (RELIEF-Studie), wird eine noch bessere Einschätzung des Einflusses von Ruxolitinib auf die konstitutionellen Symptome erwartet. Eine aktuell in Deutschland aktive Studie vergleicht Ruxolitinib mit BAT bei PV und ET (Ruxo-BEATStudie, EudraCT 2013-002132-25) und schließt auch nicht vorbehandelte Patienten ein.

Weitere TKI (CYT387-Momelotinib, LY2784544) wurden in klinischen Phase-I-/-II-Studien untersucht (NCT1998828 bzw. NCT01134120). Klinische Studien wurden bei MPN auch mit HistonDeacetylase-Inhibitoren durchgeführt. Bei Patienten $(n=44)$, die gegenüber HU alleine resistent waren, wurde die Kombination von HU und Givinostat im Rahmen eine Phase-II-Studie untersucht. In etwa der Hälfte der Patienten wurde eine komplette oder partielle Remission erzielt, was die Wirksamkeit dieser Substanz bei der PV belegt [52]. In weiteren laufenden Studien wird IFN mit HUinderPrimärtherapieverglichen(NCT01259856 und NCT01949805). Erste Beobachtungen lassen positive Effekte der Kombination von Ruxolitinib mit niedrig dosiertem IFN erkennen [53].

\section{Klinische Relevanz \\ Der JAK1/2-Tyrosinkinaseinhibitor Ruxolitinib zeigte in einer klinischen Studie eine gute Kontrolle der gesteigerten Myeloproliferation und der vielfältigen klinischen Symptome und ist für die Behandlung von Patienten mit PV, die gegenüber Hydroxyurea intolerant oder resistent sind, zugelassen.}

\section{Ausblick}

Im vergangenen Jahrzehnt wurden eindrucksvolle Fortschritte hinsichtlich des Verständnisses der Pathogenese, der Diagnostik und der Entwicklung von neuen zielgerichteten Therapien bei der PV und anderen MPN gemacht. Mit Ruxolitinib steht erstmals ein TKI für die Behandlung einer Subgruppe von Patienten mit PV zur Verfügung. Weitere Daten werden benötigt, die es erlauben die Wirksamkeit von Ruxolitinib bei der Gesamtheit der PV-Patienten einzuschätzen und die Langzeiteffekte zu bewerten. IFN ist bei PV hochwirksam und stellt derzeit die einzige Substanz dar, welche den malignen Klon signifikant reduzieren kann, was positive Effekte auf die Spätfolgen der PV erwarten läßt. Trotz dieser Fortschritte sind weitere Anstrengungen zur Therapieoptimierung der PV auch in Zukunft dringend erforderlich.

\section{Interessenkonflikt}

E. L. erklärt, dass sie Honorare der Firmen Novartis und TEVA erhalten hat. M. G. erklärt, dass er Honorare von Novartis, Shire, AOP orphan, Roche, Pfizer, Genzyme und Jansen erhalten hat. S. K. erklärt, dass er Honorare der Firmen AOP, Ariad, Baxalta, BMS, Novartis, Novartis-Stiftung, Pfizer und Shire erhalten hat.

\section{Literatur}

1 Dameshek W. Some speculations on myeloproliferative syndromes. Blood 1951; 6: 372-375

2 Rowley JD. Letter: A new consistent chromosomal abnormality in chronic myelogenous leukaemia identified by quinacrine fluorescence and Giemsa staining. Nature 1973; 243: 290-293

3 Shtivelman E, Lifshitz B, Gale RP et al. Fused transcript of abl and bcr genes in chronic myelogenous leukemia. Nature 1985; 315 : 550-554

4 James C, Ugo V, Couédic ] et al. A unique clonal JAK2 mutation leading to constitutive signaling

Vollständiges Literaturverzeichnis unter http://dx.doi.org/10.1055/s-0041-103336 
causes polycythaemia vera. Nature 2005; 434 : 1144-1148

5 Moulard O, Mehta J, Fryzek J et al. Epidemiology of myelofibrosis, essential thrombocythemia, and polycythemia vera in the European Union. Eur J Haematol 2014; 92: 289-297

6 Tefferi A, Rumi E, Finazzi G et al. Survival and prognosis among 1545 patients with contemporary polycythemia vera: an international study. Leukemia. 2013; 27: 1874-1881

7 Gruppo Italiano Studio Polycythemia vera. The natural history of 1213 patients followed for 20 years. Ann Intern Med 1995; 123: 656-664

8 Chiewitz E, Thiede T. Complications and causes of death in polycythemia vera. Acta Med Scand 1962; 172: 513-523

9 Cervantes F, Tassies D, Salgado C et al. Acute transformation in nonleukemic chronic myeloproliferative disorders: actuarial probability and main characteristics in a series of 218 patients. Acta Haematol 1991; 85: 124-127

10 Alvarez-Larrán A, Bellosillo B, Martínez-Avilés L et al. Postpolycythaemic myelofibrosis: frequency and risk factors for this complication in 116 patients. Br J Haematol 2009; 146: 504-509

11 Passasamonti F, Rumi E, Pietra D et al. A prospective study of 338 patients with polycythemia vera: the impact of JAK2 (V617F) allele burden and leukocytosis on fibrotic or leukemic disease transformation and vascular complications. Leukemia 2010; 24: 1574-1579

12 Finazzi G, Caruso V, Marchioli R et al. Acute leukemia in polycythemia vera: an analysis of 1638 patients enrolled in a prospective observational study. Blood 2005; 105: 2664-2670

13 WHO classification of tumours of haemopoietic and lymphoid tissues. WHO Press 2008: 40-43

14 Tefferi A, Thiele J, Vannucchi AM, Barbui T. An overview on CALR and CSF3R mutations and a proposal for revision of WHO diagnostic criteria for myeloproliferative neoplasms. Leukemia 2014; 28 : 1407-1413

15 Klampfl T, Gisslinger H, Harutyunyan AS et al. Somatic mutations of calreticulin in myeloproliferative neoplasms. N Engl J Med 2013; 369 : 2379-2390

16 Nangalia J, Massie CE, Baxter EJ et al. Somatic CALR mutations in myeloproliferative neoplasms with nonmutated JAK2. N Engl J Med 2013; 369: 2391-2405

17 Lengfelder E, Petrides PE, Grießhammer M. Polycythaemia vera. Empfehlungen der Fachgesellschaft zur Diagnostik und Therapie hämatologischer und onkologischer Erkrankungen 2014. www.dgho-onkopedia.de

18 Siegel FP, Petrides PE. Angeborene und erworbene Polyzythämien. Dtsch Ärztebl 2008; 105: 62-68

19 Vainchencker W, Dusa A, Constantinescu SN. JAKs in pathology: role of Janus kinases in hematopoietic malignancies and immunodeficiencies. Semin Cell Dev Biol 2008; 19: 385-393

20 O'Shea J], Schwartz DM, Villarino AV et al. The JAK-STAT pathway: impact on human disease and therapeutic intervention. Annu Rev Med 2015; 66: 311-328.

21 Lindsley RC, Ebert BL. The biology and clinical impact of genetic lesions in myeloid malignancies. Blood 2013; 122: 3741-3748

22 Lundberg P, Karow A, Nienhold R et al. Clonal evolution and clinical correlates of somatic mutations in myeloproliferative neoplasms. Blood 2014; 123: 2220-2228

23 Ortmann CA, Kent DG, Nangalia J et al. Effect of mutation order on myeloproliferative neoplasms. N Engl J Med 2015; 372: 601-612
24 Falanga a, Marcheti M. Thrombosis in myeloproliferative neoplasms. Semin Thromb Hemost 2014; 40: 348-358

25 Dentali F, Squizzato A, Brivio L. JAK2V617F mutation for the early diagnosis of $\mathrm{Ph}$ - myeloproliferative neoplasms in patients with venous thromboembolism: a meta-analysis. Blood 2009; 113: 5617-5623

26 Smalberg JH, Arends LR, Valla DC et al. Myeloproliferative neoplasms in Budd-Chiari syndrome and portal vein thrombosis: a meta-analysis. Blood 2012: 120: 4921-4928

27 Dentali F, Ageno W, Rumi E et al. Cerebral venous thrombosis and myeloproliferative neoplasms: results from two large databases. Thromb Res 2014; 134: 41-43

28 Kiladjian J], Cervantes F, Leebeek FW et al. The impact of JAK2 and MPL mutations on diagnosis and prognosis of splanchnic vein thrombosis: a report on 241 cases. Blood 2008; 111: 4922-4929

29 Scherber R, Dueck AC, Johansson P et al. The Myeloproliferative Neoplasm Symptom Assessment Form (MPN-SAF): international prospective validation and reliability trial in 402 patients. Blood 2011; 1 18: 401-408

30 Mesa R, Miller CB, Thyne M et al. Impact of Myeloproliferative Neoplasms (MPNs) on Patients' Overall Health and Productivity: Results from the MPN Landmark Survey in the United States, Blood 2014, 124: Abstract 3183

31 Barbui T, Barosi G, Birgegard G et al. Philadelphianegative classical myeloproliferative neoplasms: critical concepts and management recommendations from European LeukemiaNet. ] Clin Oncol 2011; 29: 761-770

32 Marchioli R, Finazzi G, Landolfi R et al. Vascular and neoplastic risk in a large cohort of patients with polycythemia vera. J Clin Oncol 2005; 23 2224-2232

33 Barbui T, Finazzi G, Falanga A. Myeloproliferative neoplasms and thrombosis. Blood 2013; 122: 2176-2184

34 Bonicelli G, Abdulkarim K, Mounier M et al. Leucocytosis and thrombosis at diagnosis are associated with poor survival in polycythaemia vera: a population-based study of 327 patients. $\mathrm{Br}$ J Haematol 2013; 160: 251-254

35 Geyer HL, Mesa R. Therapy for myeloproliferative neoplasms: when, which agent, and how? Blood 2014; 24: 3529-3537

36 Marchioli R, Finazzi G, Specchia G et al. CYTO-PV Collaborative Group. Cardiovascular events and intensity of treatment in polycythemia vera. $\mathrm{N}$ Engl Med 2013: 368: 22-33

37 Landolfi R, Marchioli R, Kutti J et al. Efficacy and safety of low-dose aspirin in polycythemia vera. $\mathrm{N}$ Engl J Med 2004; 350: 114-124

38 Najean Y, Dresch C, Rain JD. The very-long-term course of polycythemia: a complement to the previously published data of the polycythemia vera study group. $\mathrm{Br}$ ] Haematol 1993; 86: 233-235

39 Berk PD, Goldberg JD, Silverstein MN et al. Increased incidence of acute leukemia in polycythemia vera associated with chlorambucil therapy. N Engl J Med 1981; 304: 441-447

40 Björkholm M, Derolf AR, Hultcrantz M et al. Treatment-related risk factors for transformation to acute myeloid leukemia and myelodysplastic syndromes in myeloproliferative neoplasms. J Clin Oncol 2011; 29: 2410-2415

41 Barosi G, Birgegard G, Finazzi G et al. A unified definition of clinical resistance and intolerance to hydroxycarbamide in polycythaemia vera and primary myelofibrosis: results of a European LeukemiaNet (ELN) consensus process. $\mathrm{Br}$ J Haematol 2010; 148: 961-963 
42 Alvarez-Larrán A, Pereira A, Cervantes F et al. Assessment and prognostic value of the European LeukemiaNet criteria for clinicohematologic response, resistance, and intolerance to hydroxyurea in polycythemia vera. Blood 2012; 119: 1363-1369

43 Lengfelder E, Berger U, Hehlmann R. Interferon alpha in the treatment of polycythemia vera. Ann Hematol 2000; 79: 103-109

44 Kiladjian J], Cassinat B, Chevret S et al. Pegylated interferon-alfa-2a induces complete hematologic and molecular responses with low toxicity in polycythemia vera. Blood 2008; 112: 3065-3072

45 Quintás-Cardama A, Kantarjian H, Manshouri T et al. Pegylated interferon alfa-2a yields high rates of hematologic and molecular response in patients with advanced essential thrombocythemia and polycythemia vera. J Clin Oncol 2009; 27 : 5418-5124

46 Haanen C, Mathe G, Hayat M. Treatment of polycythemia vera by radiophosphorus or busulfan: a randomized trial. Br J Cancer 1981; 44: $75-80$

47 Kuriakose ET, Gjoni S, Wang YL et al. JAK2V617F allele burden is reduced by busulfan therapy: a new observation using an old drug. Haematologica 2013; 98: e135-137
48 Tefferi A. JAK inhibitors for myeloproliferative neoplasms: clarifying facts from myths. Blood 2012; 119: 2721-2730

49 Harrison C, Kiladjian J], Al-Ali HK et al. JAK inhibition with ruxolitinib versus best available therapy for myelofibrosis. N Engl ] Med 2012; 366: 787-798

50 Verstovsek S, Mesa RA, Gotlib J et al. A double-blind, placebo-controlled trial of ruxolitinib for myelofibrosis. N Engl J Med 2012; 366: 799-807

51 Vannucchi AM, Kiladjian J], Griesshammer $\mathrm{M}$ et al. Ruxolitinib versus standard therapy for the treatment of polycythemia vera. $\mathrm{N}$ Engl J Med 2015; 372: 426-435

52 Finazzi G, Vannucchi AM, Martinelli V et al. A phase II study of Givinostat in combination with hydroxycarbamide in patients with polycythaemia vera unresponsive to hydroxycarbamide monotherapy. Br J Haematol 2013; 161: 688-694

53 Bjørn ME, de Stricker K, Kjær L et al. Combination therapy with interferon and JAK1-2 inhibitor is feasible: Proof of concept with rapid reduction in JAK2V617F-allele burden in polycythemia vera. Leuk Res Rep 2014; 3: 73-75

Der Beitrag wurde gemäß folgendem Erratum korrigiert:

Der englische Titel des Beitrags „Diagnostik und Therapie der Polycythaemia vera im Jahre 2015“ (Dtsch Med Wochenschr 2015; 140: 1501-1506) lautet:

Diagnosis and treatment of polycythemia vera in the year 2015 